\title{
IMPLEMENTATION AND VISUALIZATION OF COMMUNITY STRUCTURE ANALYSIS USING NEO4J
}

\author{
Vaisakhan $\mathbf{A}^{1}$, Neethesh $\mathrm{N} \mathrm{Shenoy}^{2}$, Sudheep Elayidom ${ }^{3}$ \\ ${ }^{1}$ M Tech Network Computing, SOE, CUSAT \\ vaishakhan.10@gmail.com, \\ ${ }^{2} M$ Tech Network Computing, SOE, CUSAT \\ nitheshnns@gmail.com \\ ${ }^{3}$ Associate Professor SOE, CUSAT \\ sudheepelayidom@hotmail.com
}

\begin{abstract}
Many systems within the present time are often depicted in the variety of networks that contains set of nodes or vertices joined along in pairs by links or edges. Social network is the best samples of these types of system. In social network the changes are occurring chop-chop that is the social activities and interactions are dynamic chop-chop. So the nature of social networks are dynamic in nature. Community detection is basic issue that encounter during social network analysis. Here in this paper we area unit presenting a straightforward methodology for showing community structure victimization the graph visual image tool Neo4j.
\end{abstract}

Keywords: Social Networks, Community Detection and Community Structure, Neo4j

\section{INTRODUCTION}

Analyzing a complex network and extracting relevant info from these networks is an vital analysis space these days. Many analysis ways square measure adopting to realize the structure of the network and to spot the communities and their structure.

A community network consist of mainly a pair of parts: nodes/vertex-indicate the users associated within the network and edges/links-indicate however or properties through that the users square measure related to one another. A simple figure showing the community structure victimization nodes and edges is shown below.

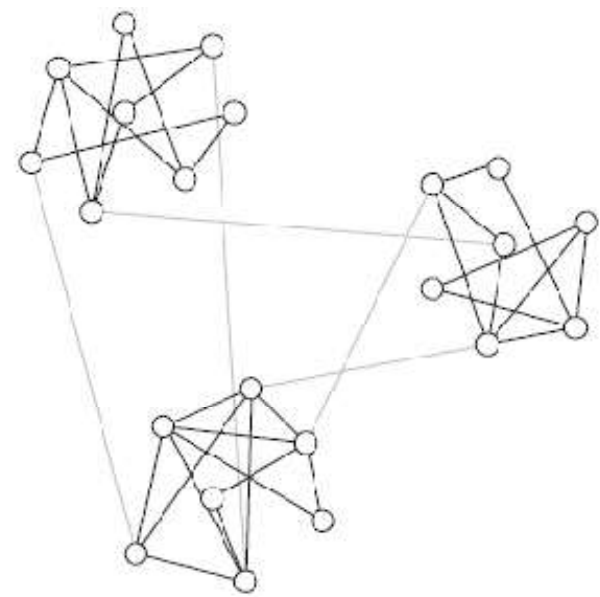

Fig 1: The above figure describes a network with 3 communities connected every different shown with gray lines. Each community contains several users delineate as nodes and they have vertices shown with dark lines.
Unfortunately, understanding the community structure in social network is very difficult since the form and structure of the network is dynamic speedily due to the user interaction and their properties. The change of properties in a social network area unit sometimes introduced by users connection in or retreating from one or a lot of teams or, by friends connecting together, or by new people creating friend with every different. This can modification the structure of the network and therefore desires reidentification of the community structure. The figure shown below tells how community structure in a network changes.

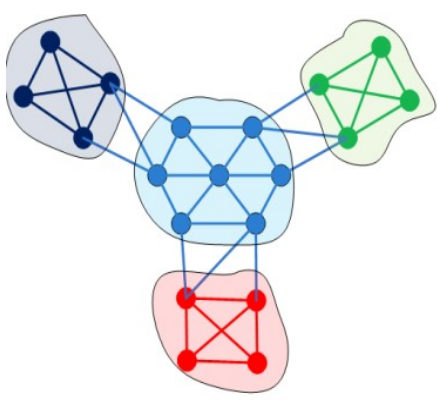

(a)

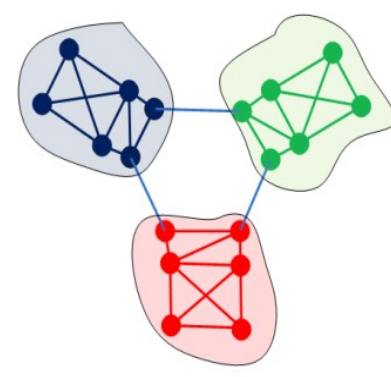

(b)
Fig 2: a) the first figure shows the first network with four communities. b) when the highest degree node is removed, the network reshapes in to a structure having 3 communities

\section{Neo4j}

Neo is a strong, scalable, high performance and embedded based computer code for economical handling of semi structured and network bound knowledge. Neo4j is a graph knowledgebase designed for network bound data. 
The relational models square measure primarily based on tables, columns and rows, Neo uses nodes, relationships and their properties. Together, all the above forms amassive network that we have a tendency to name as node house. $\mathrm{Neo} 4 \mathrm{j}$ handles semi structured data. Semi structured data has few necessary however several elective attributes. As a result, it has a awfully dynamic structure, sometimes it varies even between each single part. Data with that abundant degree of variance is tough to be handlea electronic information service however will be simply painted with the assistance of $\mathrm{Neo} 4 \mathrm{j}$ model. Neo is Associate in Nursing embedded based mostly engine, that is it is small, light weight and nonintrusive Java librarythence it will be simply enforced. Neo $4 \mathrm{j}$ has high performance and quantifiability that is it will handle $100+$ countless nodes, relationships and properties. Neo is strong. It has full support for JTA and JTS, 2PC distributed ACID transactions, and transaction recovery. Thus Neo4j will be merely outlined as

A Graph —records data in $\rightarrow$ Nodes —which have $\rightarrow$ Properties

\section{COMMUNITY STRUCTURE USING Neo4j}

For showing the community structure using Neo4j take into account a network that deals with four completely different models of Volkswagen (Polo,Vento,Jetta and Passat ). four users square measure measurement the models and every person likes and dislikes the 4 completely different models in keeping with their user expertise. Here each user likes and dislikes completely different models of the Volkswagen, using this info the community structure is fashioned.

The code for showing the community structure is shown below:

The code for the node Volkswagen model is:

CREATE (volkswagen:Manufacturer \{model : 'P', year : date' \})

Similarly for the alternative models additionally.

Code for the node user is

CREATE (x:User $\{$ name:' $x$ ' $\}$ )

Code for the property or the relation that shows between user and the car model is

CREATE (x)-[:Property \{ model : 'p' $\}]->$ ( volkswagen)

After execution the code in the Neo4j server the community structure is discovered

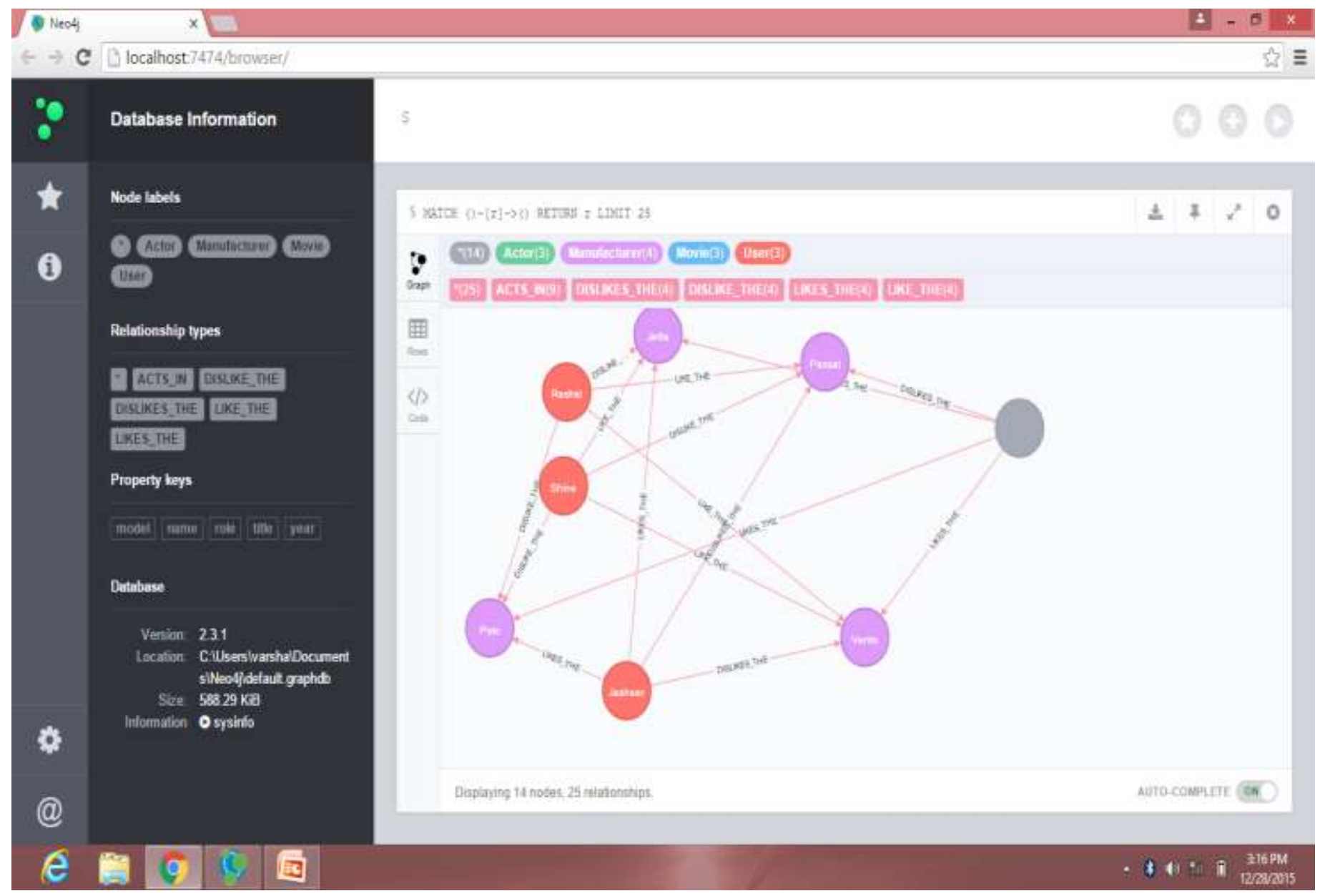


The completely different models of Volkswagen beside the four different users and therefore the properties connecting them (link) is shown because the result victimisation Neo4j.

\section{CONCLUSION}

In this paper, we have conferred the thought of community structure in social network and a unique and straightforward method of showing the community structure victimization the graph mental image tool Neo4j.

A large volume of knowledge and knowledge continues to be untouched and unexploited as a result of the dearth of knowledge modeling and management tools that aren't ready to method these data's and knowledge. Neo4j can nearly handle these knowledge since it is build from the scratch of those reasonably setting. If the data gift with you is of course ordered and semi structured then Neo4j is that the most elegant and versatile tool that you simply is alter.

\section{REFERENCES}

[1]. Deepjyoti Choudhury1, Arnab Paul,Community Detection in Social Networks: An Overview. IJRET: International Journal of Research in Engineering and Technology eISSN: 2319-1163 | pISSN: 2321-7308

[2]. Dipesh P. Joshi \& Kamal K Sutaria ,A Comprehensive Analysis of Social Network Mining., International Journal of Computer Science Engineering and Information Technology Research (IJCSEITR)ISSN(P): 2249-6831； ISSN(E): 22497943,Vol. 3, Issue 5, Dec 2013, 147-152.

[3]. Nam P. Nguyen, Thang N. Dinh, Ying Xuan, My T. Thai, Adaptive Algorithms for Detecting Community Structure in Dynamic Social Networks . IEEE INFOCOM 2011.

[4]. Kamal Sutaria, Dipesh Joshi, Dr.C.K.Bhensdadiya ,Kruti Khalpada, An Adaptive Approximation Algorithm for Community Detection in Social Network.IEEE International Conference 2015.

[5]. M. E. J. Newman and M. Girvan. Finding and evaluating community structure in networks. Phy. Rev. E 69, 2004.

[6]. Graph Theoretic and Spectral Analysis of Enron Email Data. Article in Computational and Mathematical Organization Theory · October 2005 Impact Factor: 0.84 - DOI: $10.1007 / \mathrm{s} 10588-005-5381-4$.

[7]. A Dynamic Algorithm For Community Detection in Social Networks.Bing Kong, Hongmei Chen, Weiyi Liu , Lihua Zhou. Proceedings of the $10^{\text {th }}$ World Congress on Intelligent Control and Automation July 68, 2012, Beijing, China.

[8]. The Neo4j Manual .http://www.neotechnology.com/

[9]. info@neodatabase.net, The Neo Database - A Technology Introduction (20061123) 